\title{
A Prospective, Randomized, Double Blind Study to Evaluate and Compare the Efficacy of Lidocaine, Granisetron and Magnesium Sulphate Pre-Medication, along with Venous Occlusion, in Attenuating the Pain caused due to Propofol Injection
} \author{
Abdullah 6 \\ ${ }^{1}$ Associate Professor, Sheri Kashmir Institute of Medical Sciences, India \\ ${ }^{2}$ Anesthesiology, Sheri Kashmir Institute of Medical Sciences, India \\ ${ }^{3}$ MD Anesthesiology, Sheri Kashmir Institute of Medical Sciences, India \\ ${ }^{4}$ Senior Resident, GMC Srinagar, India \\ ${ }^{5}$ Professor of Anesthesiology, Sheri Kashmir Institute of Medical Sciences, India \\ ${ }^{6}$ Senior Resident, Sheri Kashmir Institute of Medical Sciences, India
}

Sheikh Irshad Ahmad ${ }^{1}$, Saiqa Khan², Tantry Tariq Gani ${ }^{3 *}$, Tanveera Gani ${ }^{4}$, Imtiaz Ahmad Naqash ${ }^{5}$ and Aasif

Submission: August 12, 2018; Published: November 19, 2018

*Corresponding author: Dr. Tantry Tariq Gani, MD Anesthesiology, Sheri Kashmir Institute of Medical Sciences, India

\begin{abstract}
A total of 150 male and female, adult patients, aged between 21-51 years, belonging to ASA grade I and II, of either sex, undergoing elective surgery under general anesthesia, were taken up for the study and randomly allocated to any of the 3 study groups of 50 patients each, using sealed envelopes. Along with venous occlusion at $50 \mathrm{~mm} \mathrm{Hg}$ for all patients, Group 1 patients received lidocaine $40 \mathrm{mg}$ pre-medication; group 2 patients received granisetron $2 \mathrm{mg}$ pre-medication while group 3 patients received magnesium sulphate $2.03 \mathrm{mmol}$ pre-medication. The demographic parameters (age, sex, ASA grading and presence of co-morbidities) were comparable in all the 3 groups. All the 3 drugs showed statistically significant results in causing pain attenuation at $0,5,10,15$ and 20 seconds of propofol injection. The overall order of efficacy of the 3 study drugs on the basis of reduction in the severityof propofol injection pain was: lidocaine > Granisetron > Magnesium sulphate.
\end{abstract}

Keywords: Patient; lidocaine; propofol; Granisetron; Magnesium sulphate

\section{Introduction}

The exact mechanism of propofol injection pain is not known. The immediate vascular pain on propofol injection is attributed to a direct irritant effect of the drug, by stimulation of venous polymodal nociceptive receptors or free nerve endings [1,2]. The delayed pain of propofol injection has an onset latency of 10-20 seconds and is proposed to be caused by an indirect effect via activation of kallikrein- kinin cascade [3,4].

A multitude of interventions have been tried for the attenuation of pain caused due to propofol injection. In the pharmacological class of interventions, several classes of drugs like alpha 2 agonists dexmeditomidine and clonidine, antiemetics- Metoclopramide, ondansetron and granisetron, barbiturates, benzodiazipines, cholinesterase inhibitors, kallikrein inhibitor-Nafamostat mesilate, NMDA receptor antagonists- ketamine and Magnesium Sulphate, nitroglycerine, NSAIDs, opioids- tramadol, pethidine, alfentanyl, sufentanyl, remifentanyl, steroids dexamethasone and hydrocortisone, local anesthetics- prilocaine and lidocaine have been tried.

In our study we studied the effect and compared the efficacy of lidocaine, which is a commonly used drug for attenuating propofol injection pain response, with magnesium sulphate and granisetron which are upcoming

drugs only recently employed for this purpose. This was done in combination with use of tourniquet which has been seen to increase the local concentration of the drugs and hence aid in their action.

\section{Materials and Methods}

This prospective, randomized, double blind study was conducted in the Department of Anesthesiology and Critical Care 
at Sher-i-Kashmir Institute of Medical Sciences (SKIMS) Soura, Kashmir (which is a tertiary care institute with an official bed capacity of 689 beds) from June 2013 to May 2015, after approval of the study protocol by the SKIMS Review Board. A total of 150 patients, belonging to American Society of Anesthesiologists (ASA) Grade I and II, of either sex, aged between 21 and 51 years, undergoing elective surgery under general anesthesia, were taken up for the study.

Patients $<21$ yrs and $>51$ yrs, belonging to ASA Grade III and IV, with history of drug allergy to propofol, lidocaine, Magnesium Sulphate and Granisetron, on medication with pain modifying drugs, Pregnant women and morbidly obese patients, scheduled for emergency surgery, with neurological deficits or Psychiatric disorders, Un-cooperative patients, having chronic pain, cardiac conduction defects and Patients on anti arrhythmic medications were excluded from the study. A written, informed consent was taken from all the patients. Patients were randomly allocated, for receiving the study drug, into 3 groups of 50 patients each. Randomization was done by opening a sealed envelope. Group 1received $2 \mathrm{ml}$ of $2 \%$ iv lidocaine in $0.9 \%$ normal saline to make a total of $5 \mathrm{ml}$ solution, Group 2- received $2 \mathrm{mg}$ of iv. granisetron in $0.9 \%$ normal saline to make a total of $5 \mathrm{ml}$ solution and Group 3received $2.03 \mathrm{mmol}$ of magnesium sulphate in $0.9 \%$ normal saline to make a total of $5 \mathrm{ml}$ solution. Drug solutions were prepared by the co-supervisor and given to the observer who would dispense $5 \mathrm{ml}$ of study drug. This way the observer was blindfolded to the drug given to the patient. The drugs were kept at room temperature and used within 20 minutes of their constitution.

Prior to the surgery, all the patients underwent routine preanesthetic check-up. Patients were visited a day before surgery, explained the procedure and an informed consent was taken. Patients who fulfilled any of the exclusion criteria were not taken for the study. The patients were kept fasting for 6-8 hrs and pre- medicated with $0.25 \mathrm{mg}$ alprazolam orally night before surgery.

On the day of surgery, after the arrival of the patient to the operating room, ECG, NIBP, Sp02, ETCO2 and temperature monitors were connected to the patient. Intravenous access with 20 -gauge cannula, on the dorsum of non- dominant hand, was made and intravenous fluid (Normal Saline) was infused at the rate of $100 \mathrm{ml} / \mathrm{hr}$. After 2 minutes, Normal Saline infusion was stopped and arm with $\mathrm{i} / \mathrm{v}$ access was elevated and kept so for $15 \mathrm{sec}$ for gravity drainage of venous blood. The procedure was again explained to the patients. No analgesic drug was given to the patient before injecting propofol. Tourniquet was applied to the forearm around 10 centimeters below the cubital fossa. Venous occlusion was done by compressing forearm with tourniquet to $50 \mathrm{mmHg}$ so as to increase the local concentration of the drug. The study drug was then injected over 20 seconds and the arm was kept occluded by tourniquet at $50 \mathrm{mmHg}$ for 60 seconds for the drug to act. After 60 seconds, occlusion was removed by deflating the tourniquet and $25 \%$ of the total calculated dose $(2 \mathrm{mg} / \mathrm{kg})$ of propofol ( $1 \% \mathrm{w} / \mathrm{v}$ in lipid base) was injected over 20 seconds. As the drug was being administered, patient was asked about any pain that he/she felt in addition to noticing his/her facial expressions for any visible signs of pain or discomfort. The evaluation of pain was done by an anesthetist blinded to the nature of the drug used. The intensity of pain was graded using 4-point verbal rating scale (McCrirrick and Hunter pain scale) and was assessed at 0, 5, 10, 15 and 20 seconds and no further, as after 20 seconds, the patient would be under the influence of Propofol. Pain on injection of premedication, if any, was also assessed and graded using the same verbal pain scale.

Statistical analysis of the data was done using version 20 of SPSS software. Data was evaluated by Chi-Square and Fischer Exact tests. A p-value of $\leq 0.05$ was taken as statistically significant while $\mathrm{p}$-value $>0.05$ was taken as statistically non-significant.

\section{Results (Table 1-4)}

Table 1: Demographic parameters like age, sex, ASA status and comorbid states between the three groups.

\begin{tabular}{|c|c|c|c|c|}
\hline \multirow{2}{*}{ Parameter } & \multicolumn{3}{|c|}{ Group } & \multirow{2}{*}{ P value } \\
\cline { 2 - 5 } & Lidocaine N=50 & Granisetron N= 50 & Mag. Sulphate N=50 & 0.123 (NS) \\
\hline Age (Mean \pm S.D) & $35.98 \pm 9.466$ & $32.70 \pm 8.988$ & $36.52 \pm 11.48$ & 0.706 (NS) \\
\hline Sex (Male/Female) & $26 / 24$ & $29 / 21$ & $25 / 25$ & 0.770 (NS) \\
\hline ASA (I/ II) & $37 / 13$ & $38 / 12$ & $40 / 10$ & 0.770 (NS) \\
\hline Comorbidity (YES/NO) & $13 / 37$ & $12 / 38$ & $10 / 40$ & \\
\hline
\end{tabular}

Table 2: Pain score experienced by patients at $0,5,10,15$ and 20 seconds of propofol injection.

\begin{tabular}{|c|c|c|c|c|c|}
\hline \multirow{2}{*}{ Pain grade } & \multicolumn{4}{|c|}{ Group } & \multirow{2}{*}{ P value } \\
\cline { 2 - 5 } & & Lidocaine N=50 & Granisetron N= 50 & Mag. Sulphate N=50 & $50(100 \%)$ \\
\hline \multirow{3}{*}{ Grade at 0 sec } & No pain & $40(80 \%)$ & $50(100 \%)$ & $0(0.0 \%)$ & $<0.001(\mathrm{~S})$ \\
\cline { 2 - 5 } & Mild pain & $8(16 \%)$ & $0(0.0 \%)$ & $0(0.0 \%)$ & $0(0.0 \%)$ \\
\cline { 2 - 5 } & Moderate pain & $2(4 \%)$ & $0(0.0 \%)$ & $41(82 \%)$ & $<0.001(\mathrm{~S})$ \\
\cline { 2 - 5 } Grade at 5 sec & Severe pain & $0(0.0 \%)$ & $0(0.0 \%)$ & $9(18 \%)$ & \\
\cline { 2 - 5 } & No pain & $48(96 \%)$ & $0(0.0 \%)$ & \\
\hline
\end{tabular}




\section{Academic Journal of Pediatrics \& Neonatology}

\begin{tabular}{|c|c|c|c|c|c|}
\hline & Moderate pain & $0(0.0 \%)$ & $3(6 \%)$ & $0(0.0 \%)$ & \\
\hline \multirow{5}{*}{ Grade at $10 \mathrm{sec}$} & Severe pain & $0(0.0 \%)$ & $0(0.0 \%)$ & $0(0.0 \%)$ & \multirow{5}{*}{$0.004(S)$} \\
\hline & No pain & $46(92 \%)$ & $35(70 \%)$ & $43(86 \%)$ & \\
\hline & Mild pain & $4(8 \%)$ & $7(14 \%)$ & $4(8 \%)$ & \\
\hline & Moderate pain & $0(0.0 \%)$ & $8(16 \%)$ & $1(2 \%)$ & \\
\hline & Severe pain & $0(0.0 \%)$ & $0(0.0 \%)$ & $2(4 \%)$ & \\
\hline \multirow{4}{*}{ Grade at $15 \mathrm{sec}$} & No pain & $50(100 \%)$ & $32(64 \%)$ & 47 (94\%) & \multirow{4}{*}{$<0.001(\mathrm{~S})$} \\
\hline & Mild pain & $0(0.0 \%)$ & $13(26 \%)$ & $0(0.0 \%)$ & \\
\hline & Moderate pain & $0(0.0 \%)$ & $3(6 \%)$ & $0(0.0 \%)$ & \\
\hline & Severe pain & $0(0.0 \%)$ & $2(4 \%)$ & $3(6 \%)$ & \\
\hline \multirow{4}{*}{ Grade at $20 \mathrm{sec}$} & No pain & $46(92 \%)$ & $38(76 \%)$ & 47 (94\%) & \multirow{4}{*}{$0.006(\mathrm{~S})$} \\
\hline & Mild pain & $4(8 \%)$ & $3(6 \%)$ & $0(0.0 \%)$ & \\
\hline & Moderate pain & $0(0.0 \%)$ & $6(12 \%)$ & $2(4 \%)$ & \\
\hline & Severe pain & $0(0.0 \%)$ & $3(6 \%)$ & $1(2 \%)$ & \\
\hline
\end{tabular}

Table 3: Comparison of 3 groups with respect to pain at different time intervals.

\begin{tabular}{|c|c|c|c|c|c|c|c|}
\hline Time & Parameter & Lidocaine & Granisetron & Mag.Sulphate & $\begin{array}{l}\text { Lidoca ine v/s } \\
\text { Granisetron }\end{array}$ & $\begin{array}{c}\text { Lidocaine } \mathrm{v} / \mathrm{s} \\
\mathrm{MgSO}_{4}\end{array}$ & $\begin{array}{c}\text { Granisetron v/s } \\
\mathrm{MgSO}_{4}\end{array}$ \\
\hline \multirow{2}{*}{$0 \mathrm{Sec}$} & Subjects with No Pain & 40 & 50 & 50 & \multirow{2}{*}{0.001} & \multirow{2}{*}{0.001} & \multirow{2}{*}{0.001} \\
\hline & Subjects with Pain & 10 & 0 & 0 & & & \\
\hline \multirow{2}{*}{$5 \mathrm{Sec}$} & Subjects with No Pain & 48 & 47 & 41 & \multirow{2}{*}{1} & \multirow{2}{*}{0.025} & \multirow{2}{*}{0.065} \\
\hline & Subjects with Pain & 2 & 3 & 9 & & & \\
\hline \multirow{2}{*}{$10 \mathrm{Sec}$} & Subjects with No Pain & 46 & 35 & 43 & \multirow{2}{*}{0.005} & \multirow{2}{*}{0.338} & \multirow{2}{*}{0.053} \\
\hline & Subjects with Pain & 4 & 15 & 7 & & & \\
\hline \multirow{2}{*}{$15 \mathrm{Sec}$} & Subjects with No Pain & 50 & 32 & 47 & \multirow{2}{*}{$<0.001$} & \multirow{2}{*}{0.042} & \multirow{2}{*}{$<0.001$} \\
\hline & Subjects with Pain & 0 & 18 & 3 & & & \\
\hline \multirow{2}{*}{$20 \mathrm{Sec}$} & Subjects with No Pain & 46 & 38 & 47 & \multirow{2}{*}{0.029} & \multirow{2}{*}{1} & \multirow{2}{*}{0.012} \\
\hline & Subjects with Pain & 4 & 12 & 3 & & & \\
\hline
\end{tabular}

Table 4: Pain caused by pre-medication i.e the study drug.

\begin{tabular}{|c|c|c|c|c|}
\hline \multirow{2}{*}{ Pain caused by premedication } & \multicolumn{3}{|c|}{ Group } & $P$ value \\
\hline & Lidocaine $n=50$ & Granisetron $n=50$ & Mag.Sulphate $n=50$ & \multirow{5}{*}{$<0.001(\mathrm{~S}$} \\
\hline No pain & $50(100 \%)$ & $48(96 \%)$ & $12(24 \%)$ & \\
\hline Mild pain & $0(0.0 \%)$ & $0(0.0 \%)$ & $19(38 \%)$ & \\
\hline Moderate pain & $0(0.0 \%)$ & $1(2 \%)$ & $15(30 \%)$ & \\
\hline Severe pain & $0(0.0 \%)$ & $1(2 \%)$ & $4(8 \%)$ & \\
\hline
\end{tabular}

\section{Discussion}

Pain on injection is the most common and troublesome side effect of propofol, associated with high recall rates even in the post-op period [5]. To resolve this issue of propofol pain, several studies have been done to find out a possible measure to prevent this pain. During the initial experience of working with propofol, the incidence of pain caused by propofol injection was reported to range from $28-90 \%$ in adults if a vein on the dorsum of hand was used [6,7]. Since then, hundreds of studies and several metaanalyses of those studies have been carried out, which have suggested many physical strategies and drugs, used alone or in combination with mechanical measures, to reduce the incidence and severity of propofol injection pain.

In order to address this issue, we conducted a prospective, randomized, double blind study titled "A prospective, randomized, double blind study to evaluate and compare the efficacy of lidocaine, granisetron and magnesium sulphate pre-medication, along with venous occlusion, in attenuating the pain caused due to propofol injection", in the department of Anesthesiology and Critical Care, at the Sher-I-Kashmir institute of medical sciences (SKIMS) Soura, Kashmir, for a period of 2 years, from June 2013 to May 2015.

A 4-point verbal response scoring system was chosen in our study, rather than Visual Analog Score (VAS), for assessment of pain, as it is reproducible, very simple and convenient to use, both for the investigator as well as the patient. Moreover, VAS assessment might not be possible with the rapidly changing state of consciousness during induction with propofol. 
In our study, subjects were comparable in their demographic profile, there being no significant intergroup differences amongst the 3 groups with reference to age, gender, ASA grading and presence or absence of co-morbities. Analysis of pain due to propofol injection and its attenuation by the pre-medication drugs along with venous occlusion, was studied at intervals of $0,5,10,15$ and 20 seconds.

At 0 seconds of injecting propofol $80 \%$ of patients $(n=40)$ experienced no pain in the lidocaine group while in the granisetron and magnesium sulphate groups, $100 \%$ of patients $(\mathrm{n}=0)$ experienced no pain. In our trial, study of pain at 0 seconds of propofol injection showed statistically significant results in all 3 groups $(\mathrm{p}<0.001)$. Intergroup comparison showed highly significant statistical difference between lidocaine and granisetron groups $(\mathrm{p}<0.001)$ and lidocaine and magnesium sulphate groups $(p<0.001)$. However, there was no significant difference in the pain attenuation between granisetron and magnesium sulphate groups $(\mathrm{p}=1.00)$, both of which caused $100 \%$ attenuation of propofol injection pain. Out of the $20 \%$ patients experiencing pain in the lidocaine group, $16 \%$ had mild pain, only $4 \%$ had moderate pain while none of the patients had severe pain.

At 5 seconds of propofol injection, $96 \%$ patients $(n=48)$ in lidocaine group, $94 \%$ patients $(n=47)$ in granisetron group and $82 \%$ patients $(n=41)$ in magnesium sulphate group experienced no pain. The study of pain at 5 seconds showed statistically significant results in all three groups $(\mathrm{p}<0.001)$. The inter-group comparisons showed statistically significant difference between the lidocaine and magnesium sulphate groups $(\mathrm{p}=0.025)$, lidocaine proving to be superior to magnesium sulphate in attenuation of pain caused due to propofol injection. Although higher percentage of patients in granisetron group (94\%) remained pain free, as compared to magnesium sulphate group (82\%), the comparison of efficacies of granisetron and magnesium sulphate groups showed statistically non-significant results. Comparison of lidocaine and granisetron groups revealed a non-significant co-relation, both being equally effective in attenuating the pain caused due to propofol injection at 5 seconds. Important thing to be noted is that all the patients experiencing pain in magnesium sulphate group (18\%), had severe degree of pain while all the patients experiencing pain in the lidocaine group (4\%), had only mild pain. Patients in the granisetron group $(6 \%)$ experienced moderate degree of pain at 5 seconds.

At 10 seconds of propofol injection, 92\% patients $(n=46)$ in lidocaine group, $70 \%$ patients $(n=35)$ in granisetron group and $86 \%$ patients $(n=43)$ in the magnesium sulphate group experienced no pain. The study of pain at 10 seconds showed statistically significant results in all 3 groups $(p=0.004)$. The intergroup statistical comparisons showed highly significant difference between the lidocaine and granisetron groups $(p=0.005)$, lidocaine proving to be far superior to granisetron in attenuation of pain caused due to propofol injection at 10 seconds. The comparison of lidocaine and magnesium sulphate groups showed statistically non-significant results $(\mathrm{p}=0.338)$, both being equally effective in attenuating propofol pain at 10 seconds of injection. Comparison of granisetron and magnesium sulphate groups, revealed a comparable but statistically non-significant relation between their efficacies in attenuating propofol pain at 10 seconds $(\mathrm{p}=0.053)$, even though magnesium sulphate showed better results than granisetron as per the percentage of patients feeling propofol pain (14\% in magnesium sulphate group v/s 30\% in granisetron group). Although the incidence of pain in lidocaine and magnesium sulphate groups was comparable but the severity of pain differed greatly in all the 3 groups, with patients in lidocaine group who experienced pain (8\%), had only mild pain, patients in granisetron group who experienced pain (30\%), had both mild (14\%) and moderate pain (16\%) while patients in magnesium sulphate group who experienced pain $(14 \%)$ had severe pain $(4 \%)$ in addition to mild (8\%) and moderate pain $(2 \%)$.

At 15 seconds of propofol injection, $100 \%$ patients of lidocaine group, $64 \%$ patients in granisetron group and $94 \%$ patients in magnesium sulphate group experienced no pain. The study of pain at 15 seconds showed statistically significant results in all 3 groups $(\mathrm{p}<0.001)$. The intergroup statistical comparisons showed highly significant difference between the results of lidocaine and granisetron groups $(p<0.001)$, lignocaine again proving to be of far superior efficacy than granisetron in attenuation of propofol injection pain at 15 seconds. The comparison of lidocaine and magnesium sulphate groups shows statistically significant results $(\mathrm{p}=0.042)$. The comparison between granisetron and magnesium sulphate groups shows statistically highly significant degree of co-relation $(\mathrm{p}<0.001)$, magnesium sulphate proving to be much better than granisetron in attenuation of propofol pain at 15 seconds of injection. However, all of the patients having pain in the magnesium sulphate group had severe pain while majority of patients in granisetron group experienced mild pain (26\%) although the incidence of pain was high (36\%).

At 20 seconds of propofol injection, 92\% patients $(n=46)$ of lidocaine group, $76 \%$ patients $(n=38)$ of granisetron group and $94 \%$ patients $(n=47)$ of magnesium sulphate group experienced no pain on injection of propofol. The study of pain at 20 seconds showed statistically significant results in all 3 groups $(\mathrm{p}=0.006)$.

The intergroup comparisons showed statistically significant difference between the lidocaine and granisetron groups $(p=0.029)$, lidocaine being superior to granisetron in attenuation of propofol pain at 20 seconds of injection. The comparison of lidocaine and magnesium sulphate groups showed statistically insignificant results $(p=1.00)$, both showing equal efficacy. The comparison between granisetron and magnesium sulphate groups showed statistically significant co-relation ( $\mathrm{p}=0.012)$, magnesium sulphate showing superior results than granisetron. However, all the patients who experienced pain in the lidocaine group (8\%), had mild pain only while patients in magnesium sulphate group experienced moderate (4\%) and severe pain (2\%). Out of the $24 \%$ of patients who experienced pain in the granisetron group, $6 \%$ had mild pain, $12 \%$ moderate pain and $6 \%$ had severe pain. 
The highest incidence of pain was observed at 10 seconds of propofol injection. Overall, lidocaine showed the best efficacy in attenuating propofol injection pain with lowest incidence of pain amongst the 3 groups recorded at 5, 10 and 15 seconds. In addition to reducing the incidence of pain, it also reduced its severity, with majority of patients experiencing only mild pain. Magnesium sulphate ranked 2 nd in the overall reduction of propofol pain, with lowest incidence of propofol pain amongst the 3 groups, recorded at 0 and 20 seconds of propofol injection. However, Magnesium sulphate failed in reducing the severity of pain, with a significant number of patients experiencing moderate and severe pain. Granisetron ranked 3rd in the overall attenuation of propofol pain, with lowest incidence of pain recorded at 0 seconds of propofol injection.

The results of our study are consistent with those of Alipour M et al. [8] who in their study titled "Paracetamol, Ondansetron, Granisetron, Magnesium Sulfate and Lidocaine and Reduced Propofol Injection Pain", found lidocaine (along with venous occlusion), to be the most efficacious drug in attenuating propofol pain [8]. Sedat Kaya and colleagues in their study done on 100 women, concluded that administration of lidocaine with venous occlusion for 60 seconds significantly reduced the incidence and severity of pain during the injection of propofol as compared to normal saline without tourniquet [9]. In the study of Ahsan Ahmed and colleagues, done on 82 women, granisetron $2 \mathrm{mg}$ along with venous occlusion for 1 minute, was found to be effective in reducing propofol injection pain, with the added advantage of relief from post-op nausea and vomiting [10]. Prasad and Dubey conducted a study in 2003 on 150 patients. In their study, 40mg of lidocaine and $2 \mathrm{mg}$ of granisetron was injected as pre-medication in two study groups with tourniquet inflation for 2 minutes. They found that lidocaine was more effective than granisetron in attenuating propofol injection pain, which is consistent with the results of our study [11].

In a study by Turan and Memis, in 2002 on 100 patients, the effect of magnesium sulphate was compared with normal saline. They concluded from their study that magnesium sulphate, 2.48 mmol, injected 20 seconds before the administration of propofol, significantly reduced the incidence of pain caused by propofol injection and maybe useful in minimizing this common side effect [12]. In another study by Honarmand and Safavi, on 200 patients with ASA class I, II, III, the effects of magnesium sulfate, ketamine, lidocaine and saline were compared. The study showed that lidocaine was significantly better than magnesium sulphate in attenuating the pain caused due to propofol injection which is again consistent with the results of our study [13]. Shoaybi and colleagues in their study concluded that pre-treatment with magnesium sulphate did not result in any significant change of propofol injection pain in comparison with lidocaine [14].

An important finding of our study was that magnesium sulphate itself was associated with a high incidence of pain on its injection. This prompted us to assess the pain caused due to pre-medication in all the 3 groups. Pain was assessed with the same pain scale which was used for assessing pain caused due to propofol injection i.e. McCrirrick and Hunter pain scale. Magnesium sulphate caused pain in $76 \%$ of patients as compared to $4 \%$ in granisetron group and $0 \%$ in lidocaine group. This was found to be statistically highly significant $(p<0.001)$. Of the total number of patients who experienced pain on injection of magnesium sulphate, 38\% ( $n=19)$ patients had mild pain, 30\% $(n=15)$ had moderate pain while $8 \%(n=4)$ had severe pain.

We searched literature for similar results in any study and found similar reports of pain on injection of magnesium sulphate in one study by Agarwal et al. The investigators studied the effects of magnesium sulfate and lidocaine on propofol injection pain relief. They concluded that $40 \mathrm{mg}$ lidocaine and $1 \mathrm{mg}$ magnesium sulfate were equally effective in lessening the injection pain of propofol ( $42 \%$ and $30 \%$, respectively) but $31 \%$ of patients in magnesium sulfate group had pain during the injection of magnesium sulfate (before the injection of propofol). Therefore, they concluded that there was no justification for using magnesium sulphate pretreatment in attenuating pain caused due to propofol injection as it itself cause pain [15]. We found that they had used a high dose (1gm) of magnesium sulphate in their study (we in our study used $500 \mathrm{mg}$ of magnesium sulphate).

Rahimzadeh and colleagues in their study, to compare the efficacy of magnesium sulphate and ondansetron pre-treatment in attenuating propofol injection pain, used only $150 \mathrm{mg}$ of magnesium sulphate and did not encounter any issue of pain on injection of magnesium sulphate pre-medication [16]. So, it can be proposed that pain on injection of magnesium sulphate is associated with a higher dose of magnesium sulphate and lowering the dose can effectively address this issue. However, Rahimzadeh and colleagues in their study also found that lower dose of magnesium sulphate resulted in lesser attenuation of pain after first 5 seconds of propofol injection. So, the benefits of using higher dose of magnesium sulphate for attenuation of propofol injection pain, have to be weighed against its de-merits and further studies need to be done to find out the appropriate dose of magnesium sulphate for attenuating propofol injection pain without causing pain on injection.

\section{References}

1. Tan CH, Onsiong MK (1998) Pain on injection of propofol. Anaesthesia 53: 468-76.

2. Eriksson M, Englesson S, Niklasson F, Hartvig P (1997) Effect of lignocaine and $\mathrm{pH}$ on propofol-induced pain. $\mathrm{Br} \mathrm{J}$ Anaesth 78(5): 502506.

3. Briggs LP, Clarke RS, Dundee JW, Moore J, Bahar M, et al. (1981) Use of di-isopropyl phenol as main agent for short procedures. $\mathrm{Br} \mathrm{J}$ Anaesth 53(11): 1197-1202.

4. Nakane M, Iwama H (1999) A potential mechanism of propofolinduced pain on injection based on studies using nafamostat mesilate. Br J Anaesth 83(3): 397-404.

5. Johnson RA, Harper NJ, Chadwick S, Vohra A (1990) Pain on injection of propofol: methods of alleviation. Anaesthesia 45(6): 439-442. 
6. Hynynen M, Korttila K, Tammisto T (1985) Pain on iv. injection of propofol (ICI 35868) in emulsion formulation: Short communication. Acta Anaesthesiol Scand 29(6): 651- 652.

7. King SY, Davis FM, Wells JE, Murchison DJ, Pryor PJ (1992) Lidocaine for the prevention of pain due to injection of propofol. Anesth Analg $74(2): 246-249$

8. Alipour M, Tabari M, Alipour M (2014) Paracetamol, Ondansetron, Granisetron, Magnesium Sulfate and Lidocaine and Reduced Propofol Injection Pain. Iran Red Crescent Med J 16(3): e16086.

9. Kaya S, Turhanoglu S, Karaman H, Ozgün S, Basak N (2008) Lidocaine for prevention of propofol injection-induced pain: a prospective, randomized, double-blind, controlled study of the effect of duration of venous occlusion with a tourniquet in adults. Curr Ther Res 69(1): 29-35.

10. Ahmed A, Sengupta S, Das T, Rudra A, Iqbal A (2012) Pre-treatment with intravenous granisetron to alleviate pain on propofol injection: A double-blind, randomized, controlled trial. Indian J Anesth 56(2): 135138.

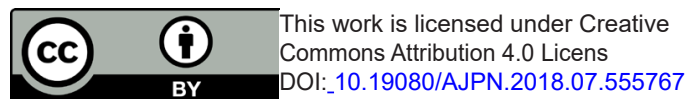

11. Dubey PK, Prasad SS (2003) Pain on injection of propofol: the effect of granisetron pretreatment. Clin J Pain 19(2): 121-124.

12. Memis D, Turan A, Karamanlioglu B (2002) The use of magnesium sulfate to prevent pain on injection of propofol. Anesth Analg 95(3): 606-608.

13. Honarmand A, Safavi M (2008) Magnesium sulphate pretreatment to alleviate pain on propofol injection: A comparison with ketamine or lidocaine. Acute Pain 10(1): 23-29.

14. Shoaybi G, Soltanimohammadi S, Rajabi M (2008) The effect of Magnesium sulfate on reducing Propofol injection pain in elective surgeries. Tehran Univ Med J 65(2): 30-34.

15. Agarwal A, Dhiraj S, Raza M, Pandey R, Pandey CK, et al. (2004) Vein pre-treatment with magnesium sulphate to prevent pain on injection of propofol is not justified. Can J Anaesth 51(2): 130-133.

16. Rahimzadeh P, Faiz SH, Nikoobakht N, Ghodrati MR (2015) Which one is more efficient on propofol $2 \%$ injection pain? Magnesium sulfate or ondansetron: A randomized clinical trial. Adv Biomed Res 4: 56.

\section{Your next submission with Juniper Publishers will reach you the below assets}

- Quality Editorial service

- Swift Peer Review

- Reprints availability

- E-prints Service

- Manuscript Podcast for convenient understanding

- Global attainment for your research

- Manuscript accessibility in different formats

( Pdf, E-pub, Full Text, Audio)

- Unceasing customer service

Track the below URL for one-step submission https://juniperpublishers.com/online-submission.php 